\title{
Calix Salophen Crown Ethers as Receptors for Neutral Molecules
}

\author{
Arjen M. Reichwein, ${ }^{a}$ Willem Verboom, ${ }^{a}$ Sybolt Harkema, ${ }^{b}$ Anthony L. Spek ${ }^{c}$ and \\ David N. Reinhoudt*a \\ a Laboratory of Organic Chemistry, University of Twente, PO Box 217, 7500 AE Enschede, \\ The Netherlands \\ ${ }^{b}$ Laboratory of Chemical Physics, University of Twente, PO Box 217, 7500 AE Enschede, \\ The Netherlands \\ ${ }^{c}$ Bijvoet Centre for Biomolecular Research, Crystal and Structural Chemistry, Utrecht University, \\ Padualaan 8, $3584 \mathrm{CH}$ Utrecht, The Netherlands
}

The calix [4] arene based salophen crown ethers 7 and 8 were synthesized as lipophilic carriers for neutral molecules. The X-ray structures of the $\mathrm{H}_{2} \mathrm{O}, \mathrm{CH}_{3} \mathrm{OH}$ and $\left(\mathrm{CH}_{3}\right)_{2} \mathrm{SO}$ complexes o* 8a have been determined.

Calix[4]arene (1) has attracted much attention as a useful building block ${ }^{1}$ for the synthesis of receptor molecules for both cations $^{2}$ and neutral molecules. ${ }^{3}$ The possibility selectively to functionalize either the upper or the lower rim and to control their conformation makes calix[4]arenes valuable platforms for the positioning of functional groups in space.

In view of our work on the complexation of neutral molecules by uranyl salophen crown ethers, ${ }^{4}$ it was interesting to incorporate a calix [4]arene moiety in such a metallomacrocycle. The resulting receptor molecules 7 and $\mathbf{8}$ are highly lipophilic, making them useful as carriers for urea in supported liquid membranes. ${ }^{5}$ Furthermore, they have a phenolic group on either side of the crown ether ring, which can be used for the incorporation of functional groups either as additional binding sites for the substrate molecule or for catalysing reactions of the complexed substrate.

In this paper, the synthesis of calix[4]arene-based metallomacrocycles 7 and 8 is described. The X-ray structures of the water, the methanol and the $\left(\mathrm{CH}_{3}\right)_{2} \mathrm{SO}$ complexes of $\mathbf{8 a}$ are presented.

\section{Results and Discussion}

Synthesis.-The synthesis of metallomacrocycles 7 and $\mathbf{8}$ is shown in Scheme 1. Alkylation of calix [4] arene $(1)^{6}$ with tosylates $2^{4 e}$ in $\mathrm{CH}_{3} \mathrm{CN}$ in the presence of 1 equiv. of $\mathrm{K}_{2} \mathrm{CO}_{3}$ as a base gave protected dialdehydes 3 in $60-65 \%$ yield $\uparrow$ Only the 1,3-dialkylated product was isolated, which is in agreement with the literature. ${ }^{7}$ The doublets in the ${ }^{1} \mathrm{H}$ NMR spectra of 3 at 4.37 and $3.32 \mathrm{ppm}$ for the methylene protons and around 31.0 ppm in the ${ }^{13} \mathrm{C}$ NMR spectra for the methylene-bridge carbon atoms of the calix[4]arene moiety reveal that the dialdehydes have a cone conformation. ${ }^{8}$ This conformation ascertains that both aldehyde groups are on the same side of the molecule, which is a prerequisite for cyclization to be possible.

Protected dialdehydes 3 were reductively deallylated with $\mathrm{Pd}\left(\mathrm{PPh}_{3}\right)_{4}$ and $\mathrm{HCOONHEt}_{3}$ in quantitative yield. The ${ }^{1} \mathrm{H}$ NMR spectra of 4 revealed no signals for the allyl ethers. Singlets for the 2-OH groups of the benzaldehyde moieties are observed at $10.87 \mathrm{ppm}$ in the ${ }^{1} \mathrm{H}$ NMR spectra, whereas the signals for the aldehyde groups were shifted from 10.4 (3) to 9.9 (4) $\mathrm{ppm}$ in the ${ }^{1} \mathrm{H}$ and from around $190(3)$ to $196(4) \mathrm{ppm}$ in the ${ }^{13} \mathrm{C}$ NMR spectra.

Cyclization of the dialdehydes 4 was performed by the addition of 1 equiv. of benzene-1,2-diamine 5 or cis-cyclohexane-1,2-

† Approximately $10 \%$ of the monoalkylated calix[4]arene was obtained.
Table 1 Selected distances $(\AA)$ of the X-ray structures

\begin{tabular}{llll}
\hline & $\mathbf{8 a} \cdot 2 \mathrm{H}_{2} \mathrm{O}$ & $\mathbf{8 a} \cdot \mathrm{MeOH}$ & $\mathbf{8 a} \cdot \mathrm{Me}_{2} \mathrm{SO}$ \\
\hline $\mathrm{U}-\mathrm{O}_{\text {apical }}$ & $1.792(8)$ & $1.36(3)$ & $1.764(11)$ \\
& $1.802(8)$ & $1.85(3)$ & $1.812(11)$ \\
$\mathrm{U}-\mathrm{O}_{\text {phenolate }}$ & $2.219(9)$ & $2.25(2)$ & $2.256(12)$ \\
& $2.250(8)$ & $2.18(2)$ & $2.25 .(12)$ \\
$\mathrm{U}-\mathrm{N}_{\text {imine }}$ & $2.56(1)$ & $2.56(4)$ & $2.52 .5(15)$ \\
& $2.55(1)$ & $2.55(4)$ & $2.63 \%(15)$ \\
$\mathrm{U}-\mathrm{O}_{\text {guest }}$ & $2.556(8)$ & $2.47(3)$ & $2.378(15)$ \\
\hline
\end{tabular}

diamine 6 to a solution of 4 and 2 equiv. of $\mathrm{Ba}(\mathrm{OT})_{2}, \ddagger$ which served as a template ion, ${ }^{4 b, 9}$ in THF. Addition of $\mathrm{CO}_{2}(\mathrm{OAc})_{2}$. $2 \mathrm{H}_{2} \mathrm{O}$ gave the crude uranyl salophen crown ethe:s 7 and 8 . Metallomacrocycles 7 were purified by precipitation from a solution in $\mathrm{CH}_{2} \mathrm{Cl}_{2}$ with cyclohexane, $\S$ whereas salophen crown ethers 8 could be purified by column chromatography on silica gel, followed by precipitation.

In the IR spectra of metallomacrocycles 7 and $\mathbf{8}$, absorptions were observed for both the imine bonds and the uranyl cations, which revealed that cyclization and complex formation had occurred. The FAB mass spectra showed $\mathbf{M}+1$ peaks, indicating that the uranyl cations are tightly bound.

The ${ }^{1} \mathrm{H}$ NMR spectrum of $7 \mathbf{a}$ exhibits two doublets for the methylene bridge protons at 4.41 and $3.39 \mathrm{ppm}$. This shows that the calix[4]arene moiety is in the cone conformation, which positions the two remaining phenolic $\mathrm{OH}$ groups on both sides of the crown ether ring. In the ${ }^{1} \mathrm{H}$ NMR spectr $c_{\text {of }} \mathbf{8}$, the 'sidedness' which results from the presence of the cyclohexane ring is observed: two singlets are observed for the prenolic $\mathrm{OH}$ groups and either the low-field half (8a) or the high-field half (8b) of the $\mathrm{AB}$ system for the methylene bridge protons appears as two doublets. In the ${ }^{13} \mathrm{C}$ NMR spectra, only three signals are observed for the carbons of the cyclohexyl ring, indicating that the cyclohexyl ring has $C_{\mathrm{s}}$-symmetry on the NMR timescale.

$X$-Ray Analysis. - Crystals of $\mathbf{8 a} \cdot 2 \mathrm{H}_{2} \mathrm{O}$, suitable for X-ray analysis, were obtained by crystallization from hot $\mathrm{CH}_{3} \mathrm{CN}$. A PLUTON view of this structure is shown in Fig. 1.

Selected bond distances are presented in Table 1. The X-ray

\$ Barium triflate was prepared by reaction of trifluoromethanesulfonic acid with barium hydroxide in $\mathrm{MeOH}$. Evaporation of the solvent gave the product as white crystals.

$\S 7 \mathbf{b}$ could not be freed from a small amount of polymeric material. 


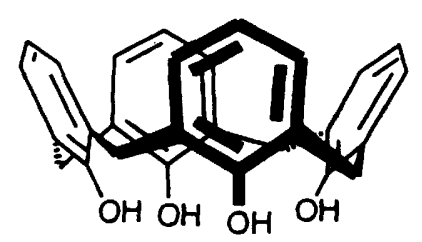

1

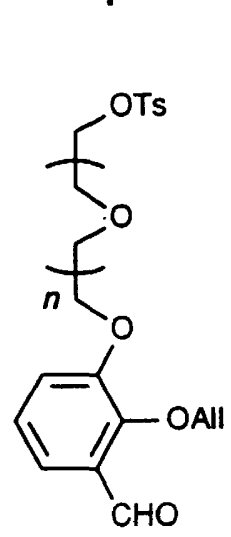

2a; $n=1$

b; $n=2$
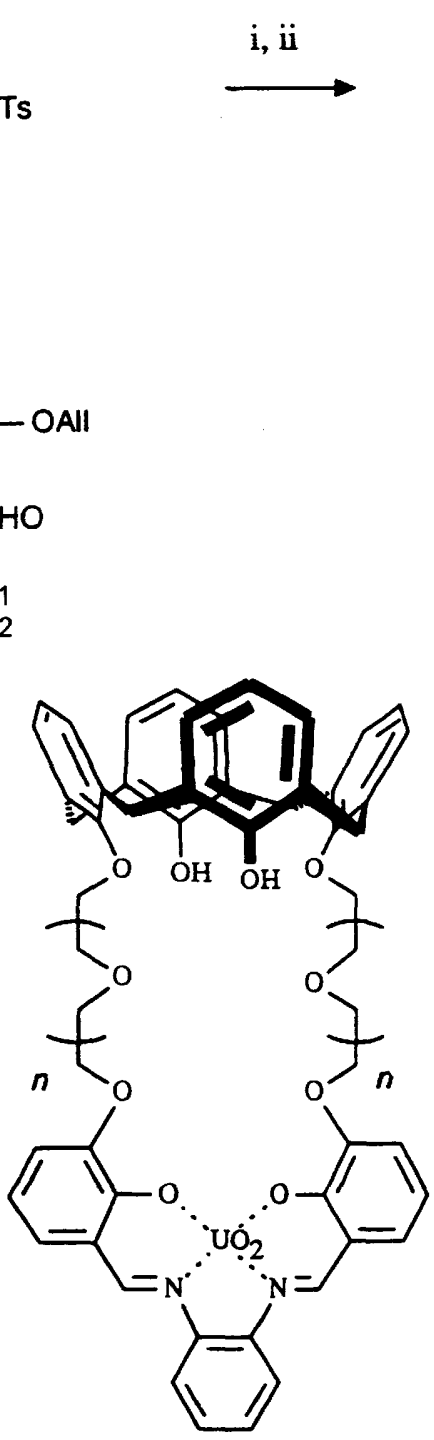

7a; $n=1(75 \%)$

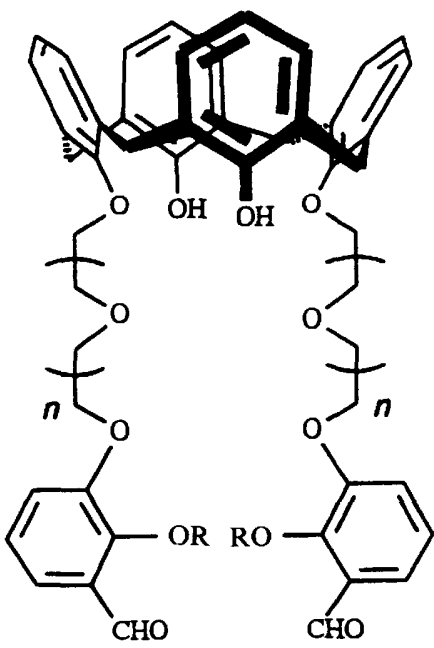

iii, iv $\begin{aligned} \text { 3a; } n=1 & (65 \%) \mathrm{R}=\text { allyl } \\ \text { b; } n=2 & (60 \%) \mathrm{R}=\text { allyl } \\ 4 \mathbf{a} ; n=1 & (100 \%) \mathrm{R}=\mathrm{H} \\ \mathrm{b} ; n=2 & (100 \%) \mathrm{R}=\mathrm{H}\end{aligned}$
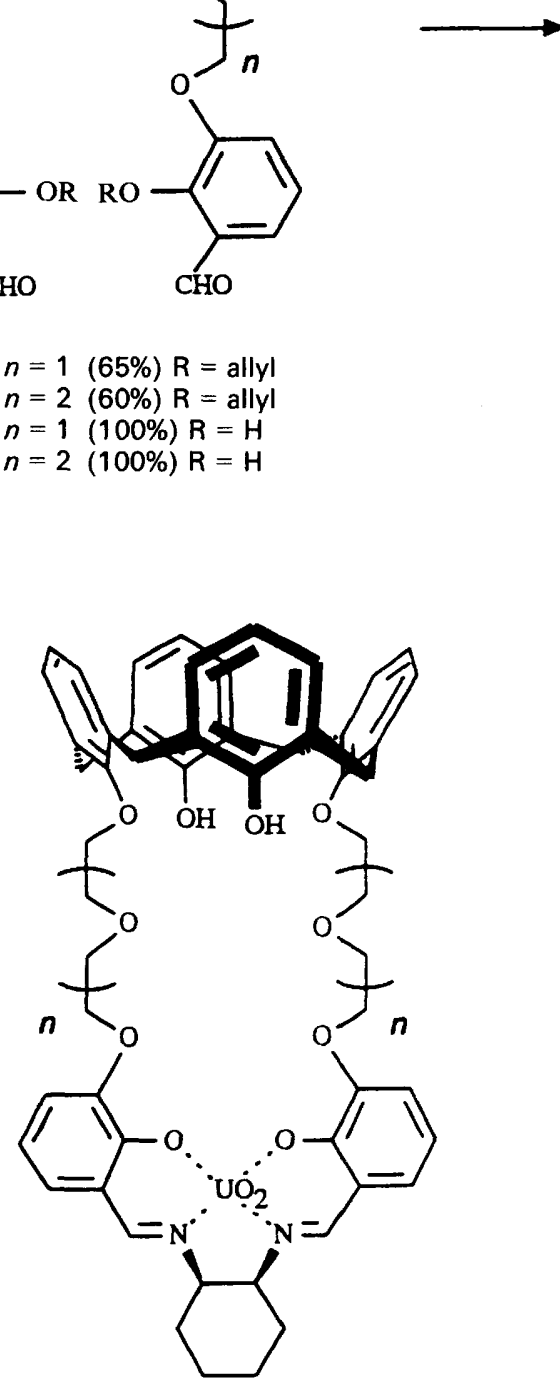

8a; $n=1 \quad(78 \%)$

b; $n=2(88 \%)$

Scheme 1 Reagents and conditions: i, $\mathrm{K}_{2} \mathrm{CO}_{3}, \mathrm{CH}_{3} \mathrm{CN}$; ii, $\mathrm{Pd}\left(\mathrm{PPh}_{3}\right)_{4}, \mathrm{HCOONHEt}_{3}$, THF-EtOH-H $\mathrm{H}_{2} \mathrm{O}$; iii, Ba(OTf $)_{2}$, benzene-1,2-diamine (5) or cis-cyclohexane-1,2-diamine (6), THF; iv, $\mathrm{UO}_{2}(\mathrm{OAc})_{2} \cdot 2 \mathrm{H}_{2} \mathrm{O}$

structure clearly shows that only one of the complexed water molecules is coordinated to the uranyl cation. The distance between the oxygen atom of the guest and the uranyl cation $(2.56 \AA)$ is slightly larger than that reported for similar $\mathrm{H}_{2} \mathrm{O}$ $(2.44 \AA),{ }^{10} \mathrm{MeOH}(2.45 \AA),{ }^{4 c, 11}$ and EtOH $(2.46 \AA)^{12}$ complexes. The second water molecule fills the cavity and is probably complexed by hydrogen bonds. The calix [4]arene moiety is in a cone conformation, which positions the two phenolic $\mathrm{OH}$ groups close to the cavity of the metallomacrocycle.

The X-ray structure of $\mathbf{8 a} \cdot \mathrm{MeOH}$, obtained by diffusion of $\mathrm{MeOH}$ into a solution of $8 \mathbf{a}$ in $\mathrm{CH}_{2} \mathrm{Cl}_{2}$, shows an
MeOH molecule coordinated to the complexed uranyl cation (Fig. 2).

Diffusion of $\mathrm{MeOH}$ into a solution of $\mathbf{8 a}$ in a mixture of $\mathrm{CH}_{2} \mathrm{Cl}_{2}$ and $\left(\mathrm{CH}_{3}\right)_{2} \mathrm{SO}$ gave crystals suitable for $\mathrm{X}$-ray analysis. Although complexes of uranyl salophen crown ethers with $\left(\mathrm{CH}_{3}\right)_{2} \mathrm{SO}$ are already known, ${ }^{3 b, c}$ this is, to the best of our knowledge, the first X-ray structure of a $\left(\mathrm{CH}_{3}\right)_{2} \mathrm{SO}$ complex (Fig. 3). The distance between the oxygen of the complexed $\left(\mathrm{CH}_{3}\right)_{2} \mathrm{SO}$ molecule and the uranyl cation $(2.38 \AA)$ is very similar to the distance between the carbonyl oxygen atom of urea and the uranyl cation in the known urea complexes $\left(2.37 \AA,,^{4 a, b} 2.36^{4 c}\right)$. 


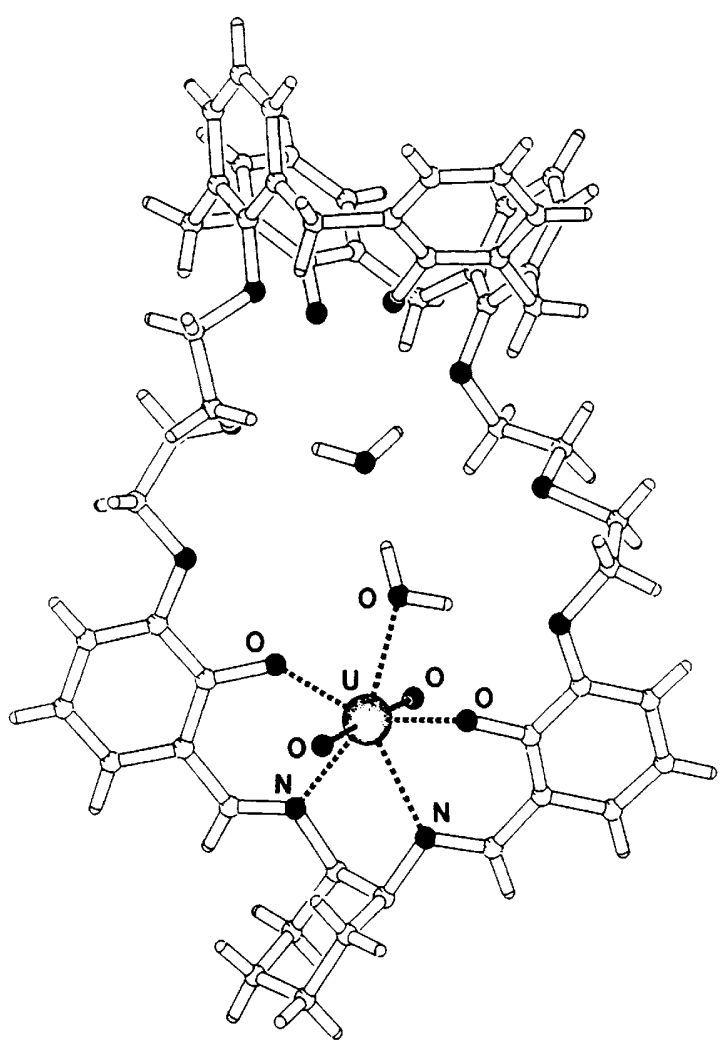

Fig. 1 X-Ray crystal structure of $8 \mathrm{a} \cdot 2 \mathrm{H}_{2} \mathrm{O}$

Extraction of Urea.*-Solid-liquid extraction experiments were performed by equilibrating a $4 \mathrm{mmol} \mathrm{dm}^{-3}$ solution of metallomacrocycles $7 \mathbf{a}$ and 8 in $\mathrm{CDCl}_{3}$ with solid urea. In all cases, significant changes in chemical shift and pattern were found for the signals of the polyether moiety.

Addition of free host to the solution of urea complex showed that a rapid exchange on the NMR timescale occurred, because only the averaged spectra were observed. The dynamic exchange made the interpretation of liquid-liquid extraction experiments at different urea concentrations $(0.05,0.1$ and 1.0 mol dm ${ }^{-3}$ ) very difficult.

The use of these receptor molecules in carrier-mediated transport of urea through a supported liquid membrane has been reported previously. ${ }^{5}$

\section{Conclusions}

A convenient synthesis of the new calix salophen crown ethers 7 and 8 has been developed. The solid-state structures of $\mathbf{8 a} \cdot 2 \mathrm{H}_{2} \mathrm{O}, \mathbf{8 a} \cdot \mathrm{MeOH}$ and $\mathbf{8 a} \cdot\left(\mathrm{CH}_{3}\right)_{2} \mathrm{SO}$ were determined showing that the guest molecules are coordinated to the uranyl cation.

\section{Experimental}

NMR spectra were recorded on a Bruker AC 250 spectrometer in $\mathrm{CDCl}_{3}$ with $\mathrm{Me}_{4} \mathrm{Si}$ as an internal standard unless stated otherwise. $J$-values are given in $\mathrm{Hz}$. Assignments of the NMR spectra are according to the numbering illustrated below. Mass spectra were obtained with a Finnigan MAT 90 spectrometer. Positive-ion fast atom bombardment (FAB) mass spectra were recorded using $m$-nitrobenzyl alcohol as the matrix. IR spectra

* Formamide and acetamide are too soluble in $\mathrm{CHCl}_{3}$ to use them in these extraction experiments: more than one equivalent of guest is extracted.

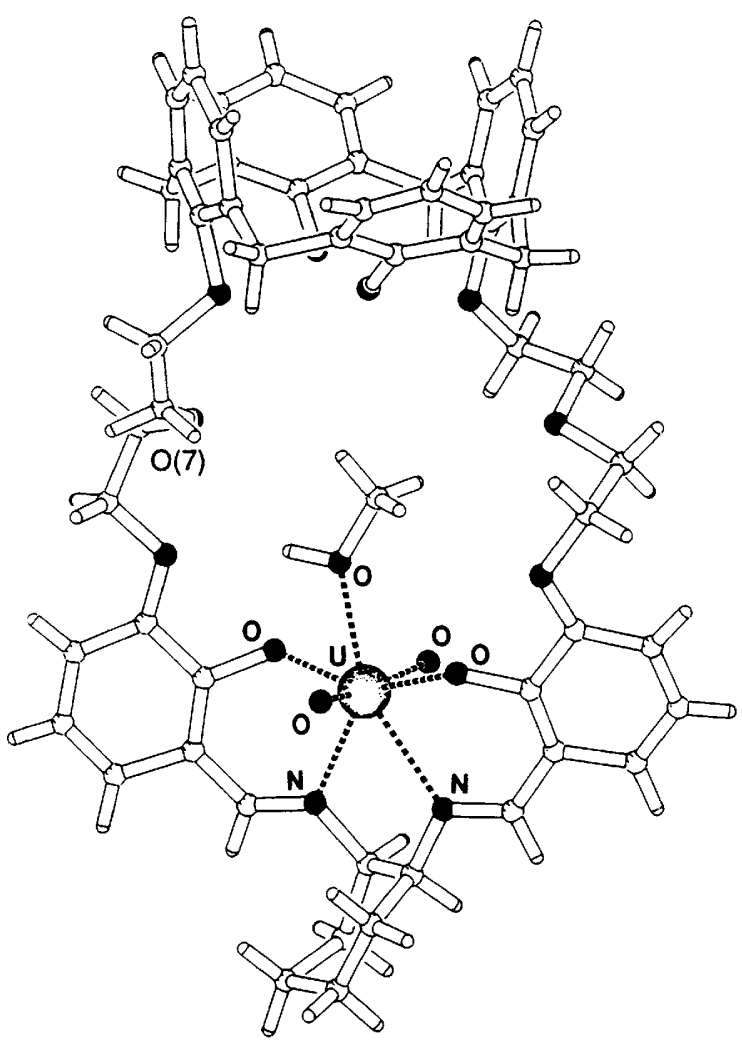

Fig. 2 X-Ray crystal structure of $8 \mathrm{a} \cdot \mathrm{MeOH}$

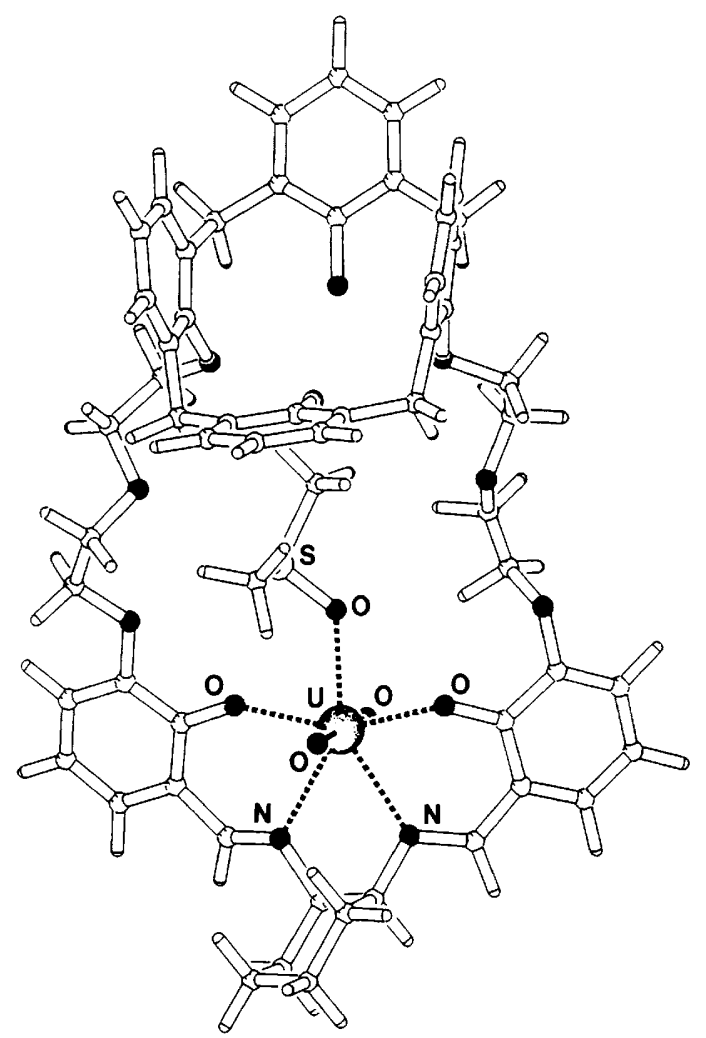

Fig. 3 X-Ray crystal structure of $8 \mathrm{a} \cdot \mathrm{Me}_{2} \mathrm{SO}$

were recorded with a Nicolet SCX FT spectrophotometer. Melting points were determined using a Reichert melting point apparatus and are uncorrected. Elemental analyses were carried out by use of a Model 1106 Carlo Erba Strumentazione elemental analyser. $\mathrm{CH}_{2} \mathrm{Cl}_{2}$, EtOAc and hexane were distilled 


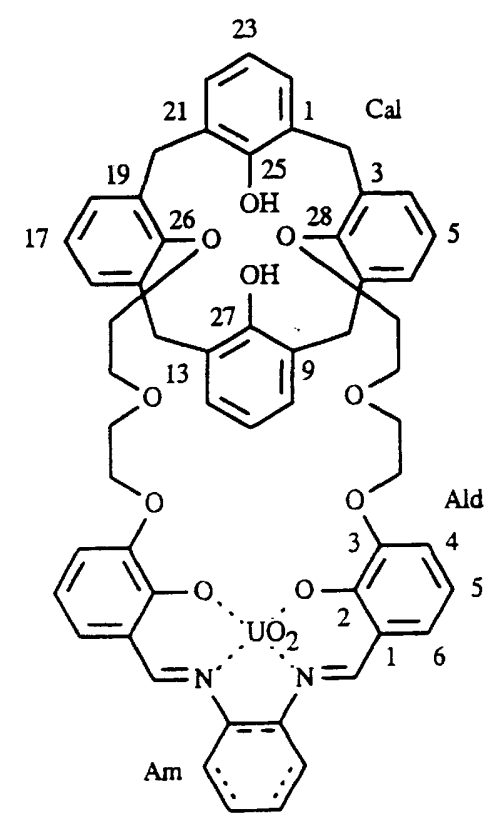

before use. $\mathrm{CH}_{2} \mathrm{CN}$ was stored over molecular sieves $(4 \AA)$ prior to use. Other chemicals were of reagent grade and were used without purification. Column chromatography was performed with silica gel (Merck: 0.040-0.063 mm). All reactions were carried out under an argon atmosphere. Calix[4]arene $1^{6}$ $(\mathrm{R}=\mathrm{H})$ was prepared according to the literature.

Care should be taken when handling uranyl-containing compounds because of their toxicity and radioactivity. ${ }^{13}$

\section{General Procedure for the Dialkylation of Calix [4] arene 1}

Synthesis of Dialdehydes 3.-A mixture of calix[4]arene 1 $(1.06 \mathrm{~g}, 2.5 \mathrm{mmol})$, tosylate $2^{4 e}(5.0 \mathrm{mmol})$, and $\mathrm{K}_{2} \mathrm{CO}_{3}(0.35 \mathrm{~g}$, $2.5 \mathrm{mmol})$ in dry $\mathrm{CH}_{3} \mathrm{CN}\left(25 \mathrm{~cm}^{3}\right)$ was refluxed for $48 \mathrm{~h}$. NaI $(0.2 \mathrm{~g})$ and $\mathrm{NEt}_{3}\left(2 \mathrm{~cm}^{3}\right)$ were added and refluxing was continued for $30 \mathrm{~min}$. The reaction mixture was cooled, diluted with $\mathrm{CH}_{2} \mathrm{Cl}_{2}\left(50 \mathrm{~cm}^{3}\right)$, and filtered through Celite. The solvent was evaporated off and the residue was redissolved in $\mathrm{CH}_{2} \mathrm{Cl}_{2}$ $\left(100 \mathrm{~cm}^{3}\right)$. The organic layer was washed with $1 \mathrm{~mol} \mathrm{dm}$ hydrochloric acid $\left(50 \mathrm{~cm}^{3}\right)$ containing a few drops of concentrated aqueous $\mathrm{NaHSO}_{3}$, dried $\left(\mathrm{MgSO}_{4}\right)$, and evaporated to dryness. After flash column chromatography of the residue, the products 3 were obtained as oils.

$3^{2}, 7^{2}$-Bis [5-(2-allyloxy-3-formylphenoxy)-3-oxapentyloxy]$1(1,3), 3(1,3), 5(1,3), 7(1,3)$-tetrabenzenacyclooctaphane $-1^{2}, 5^{2}$ diol (3a).-Eluent EtOAc-hexane $(2: 3)$, yield $65 \% ; v_{\max } / \mathrm{cm}^{-1}$ $3352(\mathrm{OH})$ and $1686(\mathrm{HC}=\mathrm{O}) ; \delta_{\mathrm{H}}\left(250 \mathrm{MHz} ; \mathrm{CDCl}_{3}\right) 3.32$ (4 H, d, $J$ 13.0, $\left.\mathrm{ArCH}_{2} \mathrm{Ar}\right), 3.9-4.0\left(8 \mathrm{H}, \mathrm{m}, \mathrm{CH}_{2} \mathrm{O}\right), 4.1-4.25$ $\left(8 \mathrm{H}, \mathrm{m}, \mathrm{CH}_{2} \mathrm{O}\right), 4.37\left(4 \mathrm{H}, \mathrm{d}, J 13.0, \mathrm{ArCH}_{2} \mathrm{Ar}\right), 4.63(4 \mathrm{H}, \mathrm{d}, J$ $\left.6.0, \mathrm{OCH}_{2} \mathrm{CH}=\mathrm{CH}_{2}\right), 5.1-5.35\left(4 \mathrm{H}, \mathrm{m}, \mathrm{OCH}_{2} \mathrm{CH}=\mathrm{CH}_{2}\right), 5.9$ $6.1\left(2 \mathrm{H}, \mathrm{m}, \mathrm{OCH}_{2} \mathrm{CH}=\mathrm{CH}_{2}\right), 6.56(2 \mathrm{H}, \mathrm{t}, J 7.5, \mathrm{CalH}), 6.65$ $(2 \mathrm{H}, \mathrm{t}, J 7.4, \mathrm{CalH}), 6.78(4 \mathrm{H}, \mathrm{d}, J 7.5, \mathrm{CalH}), 6.9-7.1(4 \mathrm{H}, \mathrm{m}$, AldC $\left._{4,4}, 5,5, \mathrm{H}\right), 7.03(4 \mathrm{H}, \mathrm{d}, J 7.4, \mathrm{CalH}), 7.35(2 \mathrm{H}$, dd, $J 7.7$ and 1.5, $\left.\mathrm{AldC}_{6,6} \cdot \mathrm{H}\right), 7.78(2 \mathrm{H}, \mathrm{s}, \mathrm{OH})$ and $10.39(2 \mathrm{H}, \mathrm{s}, \mathrm{CHO})$; $\delta_{\mathrm{C}}\left(63 \mathrm{MHz} ; \mathrm{CDCl}_{3}\right) 31.0\left(\mathrm{t}, \mathrm{ArCH}_{2} \mathrm{Ar}\right), 68.6-75.3\left(\mathrm{t}, \mathrm{CH}_{2} \mathrm{O}\right)$, $118.6 \quad\left(\mathrm{t}, \quad \mathrm{OCH}_{2} \mathrm{CH}=\mathrm{CH}_{2}\right), \quad 119.3, \quad 119.7, \quad 123.9 \quad(\mathrm{~d}$, $\left.\mathrm{AldC}_{4,4^{\prime}} 5^{\prime} 5^{\prime} 6,6^{\prime}\right), 118.9,125.2\left(\mathrm{~d}, \mathrm{CalC}_{5,11,17,23}\right), 128.4,128.8$ (d, CalC ( $\left._{4,6,10,12,16,18,22,24}\right), 129.9\left(\mathrm{~s}, \mathrm{AldC}_{1,1}\right), 127.8,132.9$ (s, $\left.\mathrm{CalC}_{1,3,7,9,13,15,19,21}\right), 133.1\left(\mathrm{~d}, \mathrm{OCH}_{2} \mathrm{CH}=\mathrm{CH}_{2}\right), 151.3,151.5$, $152.0,153.0\left(\mathrm{~s}, \mathrm{AldC}_{2,2^{\prime}, 3,3^{\prime}}\right.$ and $\left.\mathrm{CalC}_{25,26,27,28}\right)$ and $190.2 \mathrm{(d}$, $\mathrm{CHO}$ ); $m / z$ (FAB) $920.2\left(\mathrm{M}^{+}\right.$, calc. for $\left.\mathrm{C}_{56} \mathrm{H}_{56} \mathrm{O}_{12} 920.4\right)$.
$3^{2}, 7^{2}$-Bis [8-(2-allyloxy-3-formylphenoxy)-3,6-dioxaoctyloxy]-1(1,3),3(1,3),5(1,3),7(1,3)-tetrabenzenacyclooctaphane- $1^{2}$, $5^{2}$-diol (3b). -Eluent EtOAc-hexane $(1: 1)$, yield $60 \% ; v_{\max } / \mathrm{cm}^{-1}$ $3357(\mathrm{OH})$ and $1687(\mathrm{HC}=\mathrm{O}) ; \delta_{\mathrm{H}}\left(250 \mathrm{MHz} ; \mathrm{CDCl}_{3}\right) 3.33$ (4 H, d, J 13.0, $\left.\mathrm{ArCH}_{2} \mathrm{Ar}\right), 3.75-4.05\left(20 \mathrm{H}, \mathrm{m}, \mathrm{CH}_{2} \mathrm{O}\right), 4.15-4.2$ $\left(4 \mathrm{H}, \mathrm{m}, \mathrm{CH}_{2} \mathrm{O}\right), 4.39\left(4 \mathrm{H}, \mathrm{d}, J 13.0, \mathrm{ArCH}_{2} \mathrm{Ar}\right), 4.7(4 \mathrm{H}, \mathrm{dd}, J$ 6.1 and $\left.1.1, \mathrm{OCH}_{2} \mathrm{CH}=\mathrm{CH}_{2}\right), 5.2-5.4\left(4 \mathrm{H}, \mathrm{m}, \mathrm{OCH}_{2} \mathrm{CH}=\mathrm{CH}_{2}\right)$, 5.95-6.15 (2 H, m, $\left.\mathrm{OCH}_{2} \mathrm{CH}=\mathrm{CH}_{2}\right), 6.6-6.75(4 \mathrm{H}, \mathrm{m}, \mathrm{CalH})$, $6.85(4 \mathrm{H}, \mathrm{d}, J 7.5, \mathrm{CalH}), 7.0-7.15\left(4 \mathrm{H}, \mathrm{m}, \mathrm{AldC}_{4,4^{\prime}, 5,5} \cdot \mathrm{H}\right), 7.04$ $(4 \mathrm{H}, \mathrm{d}, J 7.4, \mathrm{CalH}), 7.41\left(2 \mathrm{H}, \mathrm{dd}, J 7.3\right.$ and 2.1, AldC $\left._{6,6}, \mathrm{H}\right), 7.70$ $(2 \mathrm{H}, \mathrm{s}, \mathrm{OH})$ and $10.43(2 \mathrm{H}, \mathrm{s}, \mathrm{CHO}) ; \delta_{\mathrm{C}}\left(63 \mathrm{MHz}, \mathrm{CDCl}_{3}\right)$ $31.1\left(\mathrm{t}, \mathrm{ArCH}_{2} \mathrm{Ar}\right), 68.4-75.4\left(\mathrm{t}, \mathrm{CH}_{2} \mathrm{O}\right), 118.9\left(\mathrm{t}, \mathrm{OCH}_{2} \mathrm{CH}=\right.$ $\mathrm{CH}_{2}$ ), 119.3, 119.5, 124.0 (d, $\left.\mathrm{AldC}_{4,4^{\prime}, 5,5^{\prime}, 6,6^{\prime}}\right), 119.0,125.3$ (d, $\left.\mathrm{CalC}_{5,11,17,23}\right), 128.5,128.9\left(\mathrm{~d}, \mathrm{CalC}_{4,6,10,12,16,18,22,24}\right), 130.1$

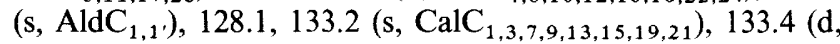
$\left.\mathrm{OCH}_{2} \mathrm{CH}=\mathrm{CH}_{2}\right), 151.5,151.8,152.1,153.2\left(\mathrm{~s}, \mathrm{AldC}_{2,2^{\prime}, 3,3^{\prime}}\right.$ and $\mathrm{CalC}_{25,26,27,28}$ ) and $190.5(\mathrm{~d}, \mathrm{CHO}) ; m / z(\mathrm{FAB}) 1008.5\left(\mathrm{M}^{+}\right.$, calc. for $\mathrm{C}_{60} \mathrm{H}_{64} \mathrm{O}_{14}$ 1008.4).

\section{General Procedure for the Deallylation of Protected Dialdehydes $\mathbf{3}$}

Synthesis of Dialdehydes 4.-A solution of 3 (2 mmol), $\mathrm{Pd}\left(\mathrm{PPh}_{3}\right)_{4}(11.5 \mathrm{mg}, 10 \mu \mathrm{mol})$, and HCOONHEt 3 (1.76 g, 12 $\mathrm{mmol})$ in a mixture of THF $\left(20 \mathrm{~cm}^{3}\right), \mathrm{EtOH}\left(20 \mathrm{~cm}^{3}\right)$ and $\mathrm{H}_{2} \mathrm{O}$ $\left(4 \mathrm{~cm}^{3}\right)$ was refluxed until the reaction had finished according to TLC (2-3 h). The solvent was evaporated off and the residue was dissolved in $\mathrm{CH}_{2} \mathrm{Cl}_{2}\left(100 \mathrm{~cm}^{3}\right)$ and washed with $1 \mathrm{~mol}$ $\mathrm{dm}^{-3}$ hydrochloric acid $\left(100 \mathrm{~cm}^{3}\right)$. The organic phase was dried $\left(\mathrm{MgSO}_{4}\right)$ and evaporated to give the products as oils in quantitative yield.

$3^{2}, 7^{2}$-Bis [5-(3-formyl-2-hydroxy) phenoxy]-3-oxapentyloxy]$1(1,3), 3(1,3), 5(1,3), 7(1,3)$-tetrabenzenacyclooctaphane- $1^{2}, 5^{2}-$ diol (4a). - $v_{\max } / \mathrm{cm}^{-1} 3380(\mathrm{OH}), 1681$ and $1655(\mathrm{HC}=\mathrm{O}) ; \delta_{\mathrm{H}^{-}}$ $\left(250 \mathrm{MHz} ; \mathrm{CDCl}_{3}\right) 3.32\left(4 \mathrm{H}, \mathrm{d}, J 13.1, \mathrm{ArCH}_{2} \mathrm{Ar}\right), 4.0-4.35$ $\left(16 \mathrm{H}, \mathrm{m}, \mathrm{CH}_{2} \mathrm{O}\right), 4.36\left(4 \mathrm{H}, \mathrm{d}, J 13.0, \mathrm{ArCH}_{2} \mathrm{Ar}\right), 6.55-6.9(10 \mathrm{H}$, $\mathrm{m}, \mathrm{ArH}), 7.02(4 \mathrm{H}, \mathrm{m}, J 7.5, \mathrm{CalH}), 7.11(4 \mathrm{H}, \mathrm{d}, J 7.9, \mathrm{CalH})$, $7.80(2 \mathrm{H}, \mathrm{s}, \mathrm{CalOH}), 9.87(2 \mathrm{H}, \mathrm{s}, \mathrm{CHO})$ and $10.86(2 \mathrm{H}, \mathrm{s}$, AldOH); $\delta_{\mathrm{C}}\left(63 \mathrm{MHz} ; \mathrm{CDCl}_{3}\right) 31.1\left(\mathrm{t}, \mathrm{ArCH}_{2} \mathrm{Ar}\right), 69.1$ $75.4\left(\mathrm{t}, \mathrm{CH}_{2} \mathrm{O}\right), 121.0\left(\mathrm{~s}, \quad \mathrm{AldC}_{1,1}\right), 119.4,120.5,124.9$ $\left(\mathrm{d}, \mathrm{AldC}_{4,4^{\prime}, 5,5^{\prime}, 6,6^{\prime}}\right), 118.9,125.3$ (d, $\left.\mathrm{CalC}_{5,11,17,23}\right), 128.4$, 128.9 (d, $\left.\mathrm{CalC}_{4,6,10,12,16,18,22,24}\right), \quad 128.0,133.2 \quad$ (s, $\mathrm{CalC}_{1,3,7,9,13,15,19,21}$ ), 147.3 (s, AldC $\left.2,2^{\prime}\right), 151.7,151.9,153.1$ (s, AldC ${ }_{3,3^{\prime}}$ and CalC $_{25,26,27,28}$ ) and 196.1 (d, CHO); $m / z$ (FAB) $840.4\left(\mathrm{M}^{+}\right.$, calc. for $\left.\mathrm{C}_{50} \mathrm{H}_{48} \mathrm{O}_{12} 840.3\right)$

$3^{2}, 7^{2}$-Bis[8-(3-formyl-2-hydroxyphenoxy)-3,6-dioxaoctyloxy]-1(1,3),3(1,3),5(1,3),7(1,3)-tetrabenzenacyclooctaphane- $1^{2}$, $5^{2}$-diol (4b). $-v_{\max } / \mathrm{cm}^{-1} 3359(\mathrm{OH}), 1681$ and $1655(\mathrm{HC}=\mathrm{O})$; $\delta_{\mathrm{H}}\left(250 \mathrm{MHz} ; \mathrm{CDCl}_{3}\right) 3.33\left(4 \mathrm{H}, \mathrm{d}, J 13.1, \mathrm{ArCH}_{2} \mathrm{Ar}\right), 3.75-4.2$ $\left(24 \mathrm{H}, \mathrm{m}, \mathrm{CH}_{2} \mathrm{O}\right), 4.40\left(4 \mathrm{H}, \mathrm{d}, J 13.0, \mathrm{ArCH}_{2} \mathrm{Ar}\right), 6.6-6.9(10 \mathrm{H}$, $\mathrm{m}, \mathrm{ArH}), 7.0-7.05(6 \mathrm{H}, \mathrm{m}, \mathrm{ArH}), 7.16(2 \mathrm{H}, \mathrm{dd}, J 7.7$ and 1.5 , AldC $\left._{6,6}, \mathrm{H}\right), 7.79$ (2 H, s, CalOH), $9.92(2 \mathrm{H}, \mathrm{s}, \mathrm{CHO})$ and 10.87 $\left(2 \mathrm{H}\right.$, br s, AldOH); $\delta_{\mathrm{C}}\left(63 \mathrm{MHz} ; \mathrm{CDCl}_{3}\right) 31.1\left(\mathrm{t}, \mathrm{ArCH}_{2} \mathrm{Ar}\right)$, 68.9-75.5 (t, $\mathrm{CH}_{2} \mathrm{O}$ ), 121.1 (s, $\mathrm{AldC}_{1,1}$ ), 119.4, 120.4, 124.7 (d, AldC $\left._{4,4^{\prime}, 5,5^{\prime}, 6,6^{\prime}}\right), 118.9,125.3\left(\mathrm{~d}, \mathrm{CalC}_{5,11,17,23}\right), 128.4,128.9(\mathrm{~d}$, $\left.\mathrm{CalC}_{4,6,10,12,16,18,22,24}\right), 128.1,133.3$ (s, CalC $\left.1,3,7,9,13,15,19,21\right)$, 147.4 (s, AldC ${ }_{2,2^{\prime}}$ ), 151.8, 152.0, 153.2 (s, AldC ${ }_{3,3^{\prime}}$ and $\left.\mathrm{CalC}_{25,26,27,28}\right)$ and $196.0(\mathrm{~d}, \mathrm{CHO}) ; m / z(\mathrm{FAB}) 928.4\left(\mathrm{M}^{+}\right.$, calc. for $\left.\mathrm{C}_{54} \mathrm{H}_{56} \mathrm{O}_{14} 928.4\right)$.

\section{General Procedure for the Cyclization of Dialdehydes 4}

Synthesis of the Calix Salophen Crown Ethers 7 and 8.-A solution of dialdehyde $4(2.5 \mathrm{mmol}), \mathrm{Ba}(\mathrm{OTf})_{2}(2.18 \mathrm{~g}, 5.0$ $\mathrm{mmol})$, and either benzene-1,2-diamine $5(270 \mathrm{mg}, 2.5 \mathrm{mmol})$ 
or cis-cyclohexane-1,2-diamine $6(285 \mathrm{mg}, 2.5 \mathrm{mmol})$ in THF $\left(250 \mathrm{~cm}^{3}\right)$ was refluxed for $30 \mathrm{~min}$. After cooling slightly, $\mathrm{UO}_{2}(\mathrm{OAc})_{2} \cdot 2 \mathrm{H}_{2} \mathrm{O}(1.59 \mathrm{~g}, 3.75 \mathrm{mmol})$ was added and refluxing was continued for about $30 \mathrm{~min}$. The solvent was evaporated off and the residue was dissolved in $\mathrm{CH}_{2} \mathrm{Cl}_{2}\left(200 \mathrm{~cm}^{3}\right)$ and washed consecutively with water $\left(2 \times 100 \mathrm{~cm}^{3}\right)$, aqueous $\mathrm{Na}_{2} \mathrm{SO}_{4}(50$ $\left.\mathrm{cm}^{3}\right)$ and water $\left(100 \mathrm{~cm}^{3}\right)$. After drying $\left(\mathrm{MgSO}_{4}\right)$ and evaporation of the solvent the crude products were obtained, which were purified by precipitation (7: $\mathrm{CH}_{2} \mathrm{Cl}_{2}$-cyclohexane) or by flash column chromatography followed by precipitation (8: eluent see below, $\mathrm{CH}_{2} \mathrm{Cl}_{2}$-cyclohexane).

$\left\{25^{2}, 28^{2}\right.$-Dihydroxy-10,11:13,14-didehydro-2,5,8,16,19,22hexaoxa-11,13-diaza-1(2,1,3),9(1,3),12(1,2),15(1,3),23(2,1,3),25$(1,3), 28(1,3)$-heptabenzenabicyclo[21.3.3]nonacosacyclophane$9^{2}, 15^{2}$-diolato $\left.(2-)-\kappa^{4} \mathrm{O}, \mathrm{O}^{\prime}, \mathrm{N}, \mathrm{N}^{\prime}\right\}$ dioxouranium (7a).-Yield $75 \%$; m.p. (from $\mathrm{CH}_{2} \mathrm{Cl}_{2}$-cyclohexane) $260-265^{\circ} \mathrm{C}$ (Found: C, $57.85 ; \mathrm{H}, 4.75 ; \mathrm{N}, 2.35 . \mathrm{C}_{56} \mathrm{H}_{50} \mathrm{~N}_{2} \mathrm{O}_{12} \mathrm{U} \cdot \mathrm{C}_{6} \mathrm{H}_{12} \cdot 2 \mathrm{H}_{2} \mathrm{O}$ requires* C, 57.23; H, 5.11; N, 2.15\%); Karl Fischer titration: Found: 3.35. Calc. for $2 \mathrm{H}_{2} \mathrm{O}: 2.77 ; v_{\max } / \mathrm{cm}^{-1} 3413(\mathrm{OH}), 1603(\mathrm{HC}=\mathrm{N})$ and $905(\mathrm{O}-\mathrm{U}-\mathrm{O}) ; \delta_{\mathrm{H}}\left(250 \mathrm{MHz} ; \mathrm{CD}_{2} \mathrm{Cl}_{2}\right) 3.39(4 \mathrm{H}, \mathrm{d}, J 13.1$, $\left.\mathrm{ArCH}_{2} \mathrm{Ar}\right), 4.1-4.35\left(12 \mathrm{H}, \mathrm{m}, \mathrm{CH}_{2} \mathrm{O}\right), 4.41(4 \mathrm{H}, \mathrm{d}, J 13.1$, $\left.\mathrm{ArCH}_{2} \mathrm{Ar}\right), 4.45-4.5\left(4 \mathrm{H}, \mathrm{m}, \mathrm{CH}_{2} \mathrm{O}\right), 6.6-6.8(6 \mathrm{H}, \mathrm{m}$, $\mathrm{AldC}_{5,5}, \mathrm{H}$ and $\left.\mathrm{CalC}_{5,11,17,23} \mathrm{H}\right), 6.92$ and $7.06(2 \times 4 \mathrm{H}, \mathrm{d}, J$ $\left.7.5, \mathrm{CalC}_{4,6,10,12,16,18,22,24} \mathrm{H}\right), 7.26$ and $7.35(2 \times 2 \mathrm{H}, \mathrm{d}, J 7.8$, $\left.\mathrm{AldC}_{4,4^{\prime}, 6,6} \mathrm{H}\right), 7.4-7.6(4 \mathrm{H}, \mathrm{m}, \mathrm{AmH}), 7.88(2 \mathrm{H}, \mathrm{s}, \mathrm{CalOH})$ and $9.37(2 \mathrm{H}, \mathrm{s}, \mathrm{HC}=\mathrm{N}) ; \delta_{\mathrm{C}}\left(63 \mathrm{MHz} ; \mathrm{CD}_{2} \mathrm{Cl}_{2}\right) 31.4(\mathrm{t}$, $\mathrm{ArCH}_{2} \mathrm{Ar}$ ), 69.9-75.0 (t, $\left.\mathrm{CH}_{2} \mathrm{O}\right), 117.1\left(\mathrm{~d}, \mathrm{AldC}_{5,5}\right.$ ), 147.2 (s, $\mathrm{AmC}_{1,1}$ ), $150.1\left(\mathrm{~s}, \mathrm{AldC}_{3,3}\right.$ ) $, 152.1,153.1$ (s, CalC $\left.25,26,27,28\right)$, $162.7\left(\mathrm{~s}, \mathrm{AldC}_{2,2}\right)$ and $165.8(\mathrm{~d}, \mathrm{HC}=\mathrm{N}) ; m / z(\mathrm{FAB}) 1181.1$ $\left([\mathrm{M}+\mathrm{H}]^{+}\right.$. Calc. for $\left.\left[\mathrm{C}_{56} \mathrm{H}_{50} \mathrm{~N}_{2} \mathrm{O}_{12} \mathrm{U}+\mathrm{H}\right] 1181.4\right)$.

$\left\{25^{2}, 28^{2}\right.$-Dihydroxy-10,11:13,14-didehydro-2,5,8,16,19,22hexaoxa-11,13-diaza-1(2,1,3),9(1,3),15(1,3),23(2,1,3),25(1,3), 28(1,3)-hexabenzena-12(1,2)-cyclohexanabicyclo[21.3.3]nonacosaphane- $9^{2}, 15^{2}$-diolato $\left.(2-) \kappa^{4} \mathrm{O}, \mathrm{O}^{\prime}, \mathrm{N}^{\prime}, \mathrm{N}^{\prime}\right\}$ dioxouranium $(\mathbf{8 a})$.Yield 78\%; m.p. (from $\mathrm{CH}_{2} \mathrm{Cl}_{2}$-cyclohexane) $255-259^{\circ} \mathrm{C}$ (Found: C, 55.1; H, 5.05; N, 2.4. $\mathrm{C}_{56} \mathrm{H}_{56} \mathrm{~N}_{2} \mathrm{O}_{12} \mathrm{U} \cdot 0.5 \mathrm{C}_{6}-$ $\mathrm{H}_{12} \cdot 2.75 \mathrm{H}_{2} \mathrm{O}$ requires $\mathrm{C}, 55.42 ; \mathrm{H}, 5.32 ; \mathrm{N}, 2.19 \%$; Karl Fischer titration: Found: 3.89. Calc. for $2.75 \mathrm{H}_{2} \mathrm{O}: 3.87 ; v_{\max } / \mathrm{cm}^{-1} 3404$ $(\mathrm{OH}), 1614(\mathrm{HC}=\mathrm{N})$ and $901(\mathrm{O}-\mathrm{U}-\mathrm{O}) ; \delta_{\mathrm{H}}\left(250 \mathrm{MHz} ; \mathrm{CD}_{2} \mathrm{Cl}_{2}\right)$ 1.65-1.9 (4 H, m, $\left.\mathrm{AmC}_{3.3}\right), 1.9-2.05$ and 2.35-2.5 (2 × $2 \mathrm{H}, \mathrm{m}$, $\left.\mathrm{AmC}_{2,2}, \mathrm{H}\right), 3.41$ (4 H, d, $J$ 13.1, $\left.\mathrm{ArCH}_{2} \mathrm{Ar}\right), 4.1-4.5(20 \mathrm{H}, \mathrm{m}$, $\mathrm{CH}_{2} \mathrm{O}$ and $\left.\mathrm{ArCH}_{2} \mathrm{Ar}\right), 4.6-4.7\left(2 \mathrm{H}, \mathrm{m}, \mathrm{AmC}_{1,1} \cdot \mathrm{H}\right), 6.6-6.85(6$ $\mathrm{H}, \mathrm{m}, \mathrm{AldC}_{5,5}, \mathrm{H}$ and $\left.\mathrm{CalC}_{5,11,17,23} \mathrm{H}\right), 6.96$ and $7.06(2 \times 4 \mathrm{H}$, d, $J$ 7.5, $\left.\mathrm{CalC}_{4,6,10,12,16,18,22,24} \mathrm{H}\right), 7.2-7.3(4 \mathrm{H}, \mathrm{m}$, AldC $\left._{4,4}, 6.6 . \mathrm{H}\right), 8.04$ and $8.09(2 \mathrm{H}, \mathrm{s}, \mathrm{OH})$ and $9.30(2 \mathrm{H}, \mathrm{s}$, $\mathrm{HC}=\mathrm{N}) ; \delta_{\mathrm{C}}\left(63 \mathrm{MHz} ; \mathrm{CD}_{2} \mathrm{Cl}_{2}\right) 22.2\left(\mathrm{t}, \mathrm{AmC}_{3,3}\right), 28.1(\mathrm{t}$, $\mathrm{AmC}_{2,2}$ ), $31.6\left(\mathrm{t}, \mathrm{ArCH}_{2} \mathrm{Ar}\right), 70.2-75.0\left(\mathrm{t}, \mathrm{CH}_{2} \mathrm{O}\right), 71.9$ (d, $\mathrm{AmC}_{1,1}$ ), $116.8\left(\mathrm{~d}, \mathrm{AldC}_{5,5^{\prime}}\right), 124.7\left(\mathrm{~s}, \mathrm{AldC}_{1,1}\right), 150.1(\mathrm{~s}$, $\left.\mathrm{AldC}_{3,3}\right), 152.3,153.3,153.4\left(\mathrm{~s}, \mathrm{CalC}_{25,26.27,28}\right), 161.3(\mathrm{~s}$, $\left.\operatorname{AldC}_{2,2}\right)$ and $167.9(\mathrm{~d}, \mathrm{HC}=\mathrm{N}) ; m / z(\mathrm{FAB}) 1187.8\left([\mathrm{M}+\mathrm{H}]^{+}\right.$. Calc. for $\left[\mathrm{C}_{56} \mathrm{H}_{56} \mathrm{~N}_{2} \mathrm{O}_{12} \mathrm{U}+\mathrm{H}\right]$ 1187.4).

$31^{2}, 34^{2}$-Dihydroxy-13,14:16,17-didehydro-2,5,8,11,19,22,25,28-octaoxa-14,16-diaza-1 $(2,1,3), 12(1,3), 18(1,3), 29(2,1,3)$, $31(1,3), 34(1,3)$-hexabenzena-15(1,2)-cyclohexanabicyclo$[27,3,3]$ pentatriacontaphane- $12^{2}, 18^{2}$-diolato $(2-) \kappa^{4} \mathrm{O}^{\prime}, \mathrm{O}^{\prime}, \mathrm{N}$,$\mathrm{N}^{\prime}$; dioxouranium (8b).-Yield $88 \%$; m.p. (from $\mathrm{CH}_{2} \mathrm{Cl}_{2}-$ cyclohexane) $202-205^{\circ} \mathrm{C}$ (Found: $\mathrm{C}, 54.9 ; \mathrm{H}, 5.15 ; \mathrm{N}, 1.95$. $\mathrm{C}_{60} \mathrm{H}_{64} \mathrm{~N}_{2} \mathrm{O}_{14} \mathrm{U} \cdot 2 \mathrm{H}_{2} \mathrm{O}$ requires $\mathrm{C}, 54.96 ; \mathrm{H}, 5.23 ; \mathrm{N}, 2.14 \%$ ).

\footnotetext{
* The presence of cyclohexane in the precipitated product was confirmed by ${ }^{1} \mathrm{H}$ NMR spectroscopy.

+ Tables of fractional atomic coordinates, bond lengths and angles have been deposited at the Cambridge Crystallographic Data Centre. See 'Instructions for Authors (1994)', in the January issue of J. Chem. Soc., Perkin Trans. 2, 1994.
}

Karl Fischer titration: Found: 2.61. Calc. for $2 \mathrm{H}_{2} \mathrm{O}: 2.75$; $v_{\max } / \mathrm{cm}^{-1} 3388(\mathrm{OH}), 1614(\mathrm{HC}=\mathrm{N})$ and $899(\mathrm{O}-\mathrm{U}-\mathrm{O}) ; \delta_{\mathrm{H}}(250$ $\left.\mathrm{MHz} ; \mathrm{CDCl}_{3}\right)$ 1.6-1.85 $\left(4 \mathrm{H}, \mathrm{m}, \mathrm{AmC}_{3,3} . \mathrm{H}\right), 1.85-2.0$ and 2.35 $2.5\left(2 \times 2 \mathrm{H}, \mathrm{m}, \mathrm{AmC}_{2,2}, \mathrm{H}\right), 3.33$ and $3.34(2 \times 2 \mathrm{H}, \mathrm{d}, J 13.0$, $\left.\mathrm{ArCH}_{2} \mathrm{Ar}\right), 4.0-4.2\left(20 \mathrm{H}, \mathrm{m}, \mathrm{CH}_{2} \mathrm{O}\right), 4.3-4.4\left(8 \mathrm{H}, \mathrm{m}, \mathrm{CH}_{2} \mathrm{O}\right.$ and $\left.\mathrm{ArCH}_{2} \mathrm{Ar}\right), 4.64 .7\left(2 \mathrm{H}, \mathrm{m}, \mathrm{AmC}_{1,1}\right), 6.6-6.75(6 \mathrm{H}, \mathrm{m}$, $\mathrm{AldC}_{5,5}, \mathrm{H}$ and $\left.\mathrm{CalC}_{5,11,17,23} \mathrm{H}\right), 6.86,7.04$ and $7.05(4+$ $\left.2 \times 2 \mathrm{H}, \mathrm{d}, J 7.5, \mathrm{CalC}_{4,6,10,12,16,18,22,24} \mathrm{H}\right), 7.2-7.3(4 \mathrm{H}, \mathrm{m}$ AldC $\left._{4,4^{\prime}, 6,6}, \mathrm{H}\right), 7.98$ and $7.99(2 \times 1 \mathrm{H}, \mathrm{s}, \mathrm{OH})$ and $9.25(2 \mathrm{H}, \mathrm{s}$, $\mathrm{HC}=\mathrm{N}) ; \delta_{\mathrm{C}}\left(63 \mathrm{MHz} ; \mathrm{CDCl}_{3}\right) 21.7\left(\mathrm{t}, \mathrm{AmC}_{3,3^{3}}\right), 27.7(\mathrm{t}$, $\mathrm{AmC}_{2,2}$ ), $31.3\left(\mathrm{t}, \mathrm{ArCH} \mathrm{H}_{2} \mathrm{Ar}\right), 70.4-75.9\left(\mathrm{AmC}_{1,1^{\prime}}\right.$ and $\left.\mathrm{CH}_{2} \mathrm{O}\right)$, $116.6\left(\mathrm{~d}, \mathrm{AldC}_{5,5^{\prime}}\right), 124.7\left(\mathrm{~s}, \operatorname{AldC}_{1,1}\right), 149.9\left(\mathrm{~s}, \mathrm{AldC}_{3,3^{3}}\right)$, $152.0,153.4\left(\mathrm{~s}, \mathrm{CalC}_{25,26,27,28}\right), 161.6\left(\mathrm{AldC}_{2,2}\right)$ and $167.6(\mathrm{~d}$, $\mathrm{CH}=\mathrm{N}) ; m / z(\mathrm{FAB}) 1275.9\left([\mathrm{M}+\mathrm{H}]^{+}\right.$, calc. for $\left[\mathrm{C}_{60^{-}}\right.$ $\left.\mathrm{H}_{64} \mathrm{~N}_{2} \mathrm{O}_{14} \mathrm{U}+\mathrm{H}\right]$ 1275.5).

\section{$X$-Ray Crystallography $\dagger$}

$X$-Ray Crystal Structure Analysis of the Water Complex of 8a.-Crystal data. $\mathrm{C}_{56} \mathrm{H}_{56} \mathrm{~N}_{2} \mathrm{O}_{10} \cdot \mathrm{UO}_{2} \cdot 4 \mathrm{CH}_{3} \mathrm{CN} \cdot 2 \mathrm{H}_{2} \mathrm{O}, M=$ 1387.4. Orange monoclinic crystals, space group $P 2_{1}, a=$ $10.374(2), b=15.062(6), c=19.613(6) \AA, \beta=101.79(2)^{\circ}$, $V=3000(3) \AA^{3}$ (average of least-squares refinement of repeated measurements of 25 reflection angles), $Z=2, d_{\text {calc }}=$ $1.54 \mathrm{~g} \mathrm{~cm}^{-3}, \mu=26.3 \mathrm{~cm}^{-1}$.

Data collection and processing. Reflections were measured on a CAD4 diffractometer, $T=110 \mathrm{~K}, \omega / 2 \theta$ scan mode, using graphite-monochromated $\mathrm{Mo}-\mathrm{K} \alpha$ radiation [scan width $(\omega)$ $(1.1+0.35 \tan \theta)^{\circ} ; 3<\theta<27.5^{\circ} ; \quad-14<h<14,0<$ $k<19 ; 0<l<23$ ]; 8475 reflections were measured of which 8016 were unique $\left(R_{\text {merge }}=2.7 \%\right.$ after an empirical absorption correction with DIFABS). ${ }^{14} \mathrm{~A}$ total of 6608 reflections with $F_{\mathrm{o}}^{2}>3 \sigma\left(F_{\mathrm{o}}^{2}\right)$ were used in the refinement.

Structure analysis and refinement. The structure was solved by Patterson methods and refined with full-matrix leastsquares. In one of the crown ether parts disorder was found for one of the carbon atoms. This could be resolved by refining two atoms with partial occupancies of $0.8 / 0.2$. The neighbouring atoms of this carbon have been refined with anisotropic thermal parameters, which showed rather large apparent thermal motion in one direction. The number of parameters refined was 362 [scale factor, extinction parameter, positional parameters of all atoms, isotropic thermal parameters, anisotropic thermal parameters for $\mathrm{U}$ and the two atoms described above; $\mathrm{H}$-atoms not included; origin in the $y$-direction fixed by the position of the U-atom]. The final $R$-factors were $R=5.5 \%, R_{\mathrm{w}}=7.5 \%$. All calculations were done with $\operatorname{SDP}^{15}$ and the illustration with PLUTON. ${ }^{16}$

$X$-Ray Crystal Structure Analysis of the Methanol Complex of 8a.-Crystal data. $\mathrm{C}_{56} \mathrm{H}_{56} \mathrm{~N}_{2} \mathrm{O}_{10} \cdot \mathrm{UO}_{2} \cdot 3 \mathrm{CH}_{4} \mathrm{O} \cdot 2 \mathrm{H}_{2} \mathrm{O}, M=$ 1319.3. Red monoclinic crystals, space group $P 2_{1} / c, a=$ 9.642(4), $b=15.785(8), c=38.01(2) \AA, \beta=93.59(5)^{\circ}, V=$ 5774(5) $\AA^{3}$ (from 19 SET4 setting angles), $Z=4, d_{\text {calc }}=$ $1.518 \mathrm{~g} \mathrm{~cm}^{-3}, \mu(\mathrm{Mo}-\mathrm{K} \alpha)=27.3 \mathrm{~cm}^{-1}$. Plate-shaped crystal $[0.10 \times 0.40 \times 0.70 \mathrm{~mm}]$.

Data collection and processing. TurboCAD4/Rotating anode diffractometer, $60 \mathrm{kV}, 150 \mathrm{~mA}, T=150 \mathrm{~K}, \omega / 2 \theta$-scan mode, graphite-monochromated Mo-K $\alpha$ radiation, $\Delta \omega=$ $(1.0+0.35 \tan \theta)^{\circ} ; 1.1<\theta<23^{\circ} ;$ hkl: 0,$8 ;-17,0 ;-41$, $41 ; 8003$ reflections scanned of which 7127 unique. The data were corrected for $\mathrm{L}_{p}$ and absorption (DIFABS). ${ }^{14}$

Structure analysis and refinement. The structure was solved with DIRDIF92 ${ }^{17}$ and refined in $F^{2}$ by full-matrix least-squares with SHELXL-93. ${ }^{18}$ Hydrogen atoms were introduced at calculated positions. Convergence was reached at $R_{1}=0.19$ $\left(w R_{2}=0.48\right), \quad S=1.05, \quad w^{-1}=\sigma^{2}\left(F^{2}\right)+\left(0.1938 \quad P^{2}\right)+$ 
909.8 $P$. The relatively high $R$ value is due to the fact that the crystals reflect poorly and with broad reflection profiles. The non-coordinating solvents are disordered and were modelled. Geometrical calculations were done with PLATON ${ }^{19}$ and the illustration with PLUTON. ${ }^{16}$ Scattering factors were taken from the International Tables. ${ }^{20}$

$X$-Ray Crystal Structure Analysis of the $\mathrm{Me}_{2} \mathrm{SO}$ Complex of 8a.-Crystal data. $\mathrm{C}_{56} \mathrm{H}_{56} \mathrm{~N}_{2} \mathrm{O}_{10} \cdot \mathrm{UO}_{2} \cdot \mathrm{C}_{2} \mathrm{H}_{6} \mathrm{SO} \cdot 3.5 \mathrm{CH}_{4} \mathrm{O} \cdot$ $3 \mathrm{H}_{2} \mathrm{O}, M=1431.42$. Orange monoclinic crystals, space group $P 2_{1} / c ; a=18.011(1), b=24.517(2), c=14.241(2) \AA, \beta=$ $107.86(1)^{\circ}, V=5985.7(8) \AA^{3}$ (from 25 SET4 setting angles in the range $\left.10<\theta<14^{\circ}\right), Z=4, d_{\text {calc }}=1.588 \mathrm{~g} \mathrm{~cm}^{-3}$, $\mu(\mathrm{Mo}-\mathrm{K} \alpha)=26.8 \mathrm{~cm}^{-1}$. Block-shaped crystal $[0.25 \times$ $0.38 \times 0.50 \mathrm{~mm}]$.

Data collection and processing. TurboCAD4/Rotating anode diffractometer, $60 \mathrm{kV}, 150 \mathrm{~mA}, T=150 \mathrm{~K}, \omega / 2 \theta$-scan mode, graphite-monochromated Mo-K $\alpha$ radiation, $\Delta \omega=$ $(0.68+0.35 \tan \theta)^{\circ} ; 1<\theta<26.5^{\circ} ; h k l:-16,17 ; 0,29 ;$ $-22,0 ; 11806$ reflections scanned of which 11104 unique. The data were corrected for $\mathrm{L}_{\mathrm{p}}$ and absorption (DIFABS). ${ }^{14} 5859$ reflections with $I>2.5 \sigma(I)$ were used in the subsequent calculations.

Structure analysis and refinement. The structure was solved with DIRDIF92 ${ }^{17}$ and refined in $F$ by full-matrix least-squares (SHELX-76). ${ }^{21}$ Hydrogen atoms were taken into account at calculated positions. Convergence was reached at $R=0.069$, $w R=0.082, w=1, S=2.06$. The $\mathrm{S}$ atom was found to be disordered over two positions $(0.75,0.25)$. The disordered solvent areas were modelled. Geometrical calculations were done with PLATON ${ }^{19}$ and the illustration with PLUTON. ${ }^{16}$ Scattering factors were taken from Cromer and Mann, ${ }^{22}$ corrected for anomalous dispersion. ${ }^{23}$

\section{References}

1 (a) C. D. Gutsche, Calixarenes, Monographs in Supramolecular Chemistry, ed. J. F. Stoddart, The Royal Society of Chemistry, Cambridge, 1989, vol. 1; (b) Calixarenes: a Versatile Class of Macrocyclic Compounds, Topics in Inclusion Science, eds. J. Vicens and V. Böhmer, Kluwer, Dordrecht, 1991, Vol. 3.

2 (a) A. Arduini, A. Pochini, S. Reverberi and R. Ungaro, J. Chem. Soc., Chem. Commun., 1984, 981; (b) R. Ungaro, A. Pochini and G. D. Andreetti, J. Inclusion Phenom. Mol. Recogn. Chem., 1984, 2, 199; (c) A. Arduini, A. Pochini, S. Reverberi and R. Ungaro, Tetrahedron, 1986, 42, 2089; (d) G. Calestani, F. Ugozzoli, A. Arduini, E. Ghidini and R. Ungaro, J. Chem. Soc., Chem. Commun., 1987, 344; (e) C. Alfieri, E. Pradi, A. Pochini, R. Ungaro and G. D. Andreetti, J. Chem. Soc., Chem. Commun., 1983, 1075; $(f)$ E. Ghidini, F. Ugozzoli, R. Ungaro, S. Harkema, A. A. El-Fadl and D. N. Reinhoudt, J. Am. Chem. Soc., 1990, 112, 6979; ( $g$ ) D. N. Reinhoudt, P. J. Dijkstra, P. J. A. in 't Veld, K.-E. Bugge, S. Harkema, R. Ungaro and E. Ghidini, $J$. Am. Chem. Soc., 1987, 109, 4761; (h) P. J. Dijkstra, J. A. J. Brunink, K.-E. Bugge, D. N. Reinhoudt, S. Harkema, R. Ungaro, F. Ugozzoli and E. Ghidini, J. Am. Chem. Soc., 1989, 111, 7567; (i) F. ArnaudNeu, E. M. Collins, M. Deasey, G. Ferguson, S. J. Harris, B. Kaitner, A. J. Lough, M. A. McKervey, E. Marques, B. L. Ruhl, M. J. SchwingWeill and E. M. Seward, J. Am. Chem. Soc., 1989, 111, 8681.
3 (a) J.-D. van Loon, J. F. Heida, W. Verboom and D. N. Reinhoudt, Recl. Trav. Chim. Pays-Bas, 1992, 111, 353; (b) J.-D. van Loon, R. G. Janssen, W. Verboom and D. N. Reinhoudt, Tetrahedron Lett., 1992, 33, 5125 .

4 (a) C. J. van Staveren, D. E. Fenton, D. N. Reinhoudt, J. van Eerden and S. Harkema, J. Am. Chem. Soc., 1987, 109, 3456; (b) C. J. van Staveren, J. van Eerden, F. C. J. M. van Veggel, S. Harkema and D. N. Reinhoudt, J. Am. Chem. Soc., 1988, 110, 4994; (c) A. R. van Doorn, R. Schaafstra, M. Bos, S. Harkema, J. van Eerden, W. Verboom and D. N. Reinhoudt, J. Org. Chem., 1991, 56, 6083; (d) A. R. van Doorn, D. J. Rushton, W. F. van Straaten-Nijenhuis, W. Verboom and D. N. Reinhoudt, Recl. Trav. Chim. Pays-Bas, 1992, 111, 421; (e) A. M. Reichwein, W. Verboom and D. N. Reinhoudt, Recl. Trav. Chim. Pays-Bas, 1993, 112, 358; $(f)$ A. M. Reichwein, W. Verboom and D. N. Reinhoudt, Recl. Trav. Chim. Pays-Bas, 1993, 112, 595 .

5(a) W. F. Nijenhuis, A. R. van Doorn, A. M. Reichwein, F. de Jong and D. N. Reinhoudt, J. Am. Chem. Soc., 1991, 113, 3607; (b) W. F. van Straaten-Nijenhuis, A. R. van Doorn, A. M. Reichwein, F. de Jong and D. N. Reinhoudt, J. Org. Chem., 1993, 58, 2265.

6 C. D. Gutsche and M. Iqbal, Org. Synth., 1990, 68, 234.

7 (a) J.-D. van Loon, A. Arduini, W. Verboom, R. Ungaro, G. J. van Hummel, S. Harkema and D. N. Reinhoudt, Tetrahedron Lett., 1990 , 30, 2681; (b) F. Bottino, L. Giunta and S. Pappalardo, J. Org. Chem., 1989, 54, 5407; (c) J.-D. van Loon, A. Arduini, L. Coppi, W. Verboom, A. Pochini, R. Ungaro, S. Harkema and D. N. Reinhoudt, J. Org. Chem., 1990, 55, 5639; (d) L. C. Groenen, B. H. M. Ruël, A. Casnati, P. Timmerman, W. Verboom, S. Harkema, A. Pochini, R. Ungaro and D. N. Reinhoudt, Tetrahedron Lett., 1991, 32, 2675.

8 C. Jaime, J. de Mendoza, P. Prados, P. M. Nieto and C. Sánchez, J. Org. Chem., 1991, 56, 3372.

9 C. J. van Staveren, D. N. Reinhoudt, J. van Eerden and S. Harkema, J. Chem. Soc., Chem. Commun., 1987, 974.

10 A. R. van Doorn, Ph.D. Thesis, University of Twente, 1992,

11 G. Bandoli, D. A. Clemente, U. Croatto, M. Vidali and P. A. Vigato, J. Chem. Soc., Dalton Trans., 1973, 2331.

12 G. Bandoli, D. A. Clemente, U. Croatto, M. Vidali and P. A. Vigato, J. Chem. Soc., Chem. Commun., 1971, 1330.

13 Dangerous Properties of Industrial Materials, 5th edn., ed. N. I. Sax, van Nostrand Reinhold, New York, 1979, pp. 1078-1079.

14 N. Walker and D. Stuart, Acta Crystallogr., Sect. A, 1983, 39, 158.

15 Structure Determination Package; B. A. Frenz and Associates Inc., College Station, TX, and Enraf-Nonius, Delft, 1983.

16 A. L. Spek, PLUTON, Program for Molecular Graphics, Utrecht University, The Netherlands, 1993.

17 P. T. Beurskens, G. Admiraal, G. Beurskens, W. P. Bosman, S Garcia-Grande, R. O. Gould, J. M. M. Smits and C. Smykalla, The DIRDIF-Program System. Technical Report of the Crystallographic Laboratory, University of Nijmegen, The Netherlands, 1992.

18 G. M. Sheldrick, SHELXL-93, Program for Crystal Structure Refinement, University of Göttingen, Germany, 1993.

19 A. L. Spek, Acta Crystallogr., Sect. A, 1990, 46, C34.

20 International Tables for Crystallography, ed. A. J. C. Wilsen, Kluwer, Dordrecht, The Netherlands, 1992, Vol. C.

21 G. M. Sheldrick, SHELX-76, Program for Crystal Structure Determination, University of Cambridge, UK, 1976.

22 D. T. Cromer and J. B. Mann, Acta Crystallogr., Sect. A, 1968, 24, 312

23 D. T. Cromer and D. Liberman, J. Chem. Phys., 1970, 53, 1891.

Paper 3/06894C

Received 18th November 1993 Accepted 7th February 1994 